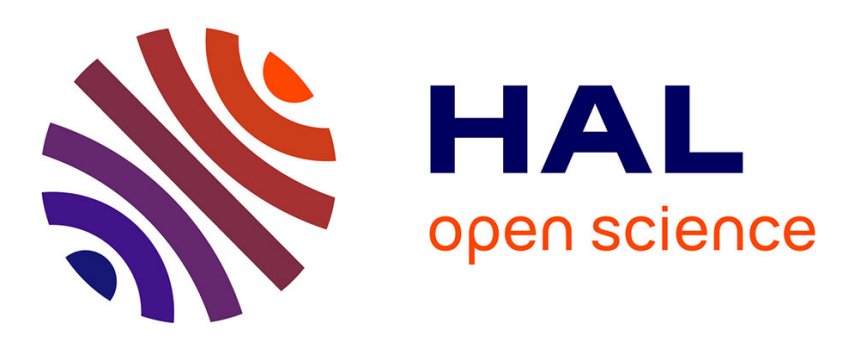

\title{
Accurate and consistent automatic seismocardiogram annotation without concurrent ECG
}

Alexandre Laurin, Farzad Khorsow, Andrew Blaber, Kouhyar Tavakolian

\section{To cite this version:}

Alexandre Laurin, Farzad Khorsow, Andrew Blaber, Kouhyar Tavakolian. Accurate and consistent automatic seismocardiogram annotation without concurrent ECG. Physiological Measurement, 2016, 37 (9), 10.1088/0967-3334/37/9/1588 . hal-01356419

\section{HAL Id: hal-01356419 \\ https://inria.hal.science/hal-01356419}

Submitted on 26 Aug 2016

HAL is a multi-disciplinary open access archive for the deposit and dissemination of scientific research documents, whether they are published or not. The documents may come from teaching and research institutions in France or abroad, or from public or private research centers.
L'archive ouverte pluridisciplinaire HAL, est destinée au dépôt et à la diffusion de documents scientifiques de niveau recherche, publiés ou non, émanant des établissements d'enseignement et de recherche français ou étrangers, des laboratoires publics ou privés. 


\title{
Accurate and consistent automatic seismocardiogram annotation without concurrent ECG
}

\author{
A Laurin ${ }^{1,2}$, F Khorsow ${ }^{3}$, A Blaber ${ }^{1}$, and Kouhyar Tavakolian Ka $^{3,4}$ \\ ${ }^{1}$ Department of Biomedical Physiology and Kinesiology, Simon Fraser University, \\ University Dr, Burnaby, BC, V5A 1S6, Canada \\ ${ }^{2}$ Inria Saclay Ile-de-France, Rue Honor d'Estienne d'Orves, Palaiseau, 91120, France \\ ${ }^{3}$ Department of Engineering, Simon Fraser University, University Dr, Burnaby, BC, \\ V5A 1S6, Canada \\ ${ }^{4}$ Electrical Engineering Department, University of North Dakota, University Ave, \\ Grand Forks, ND, 58203, USA \\ E-mail: alexandre.laurin@inria.fr
}

\begin{abstract}
Seismocardiography (SCG) is the measurement of vibrations in the sternum caused by the beating of the heart. Precise cardiac mechanical timings that are easily obtained from SCG are critically dependent on accurate identification of fiducial points.

So far, SCG annotation has relied on concurrent ECG measurements. An algorithm capable of annotating SCG without the use any other concurrent measurement was designed. We subjected 18 participants to graded lower body negative pressure. We collected ECG and SCG, obtained R peaks from the former, and annotated the latter by hand, using these identified peaks. We also annotated the SCG automatically.

We compared the isovolumic moment timings obtained by hand to those obtained using our algorithm. Mean \pm confidence interval of the percentage of accurately annotated cardiac cycles were $97.2 \pm 3.7 \%, 93.0 \pm 4.6 \%, 76.9 \pm 14.9 \%, 61.6 \pm 17.2 \%$, and $65.0 \pm 14.0 \%$ for levels of negative pressure $0,-20,-30,-40$, and $-50 \mathrm{mmHg}$.

$\mathrm{LF} / \mathrm{HF}$ ratios, the relative power of low-frequency variations to high-frequency variations in heart-beat intervals, obtained from isovolumic moments were also compared to those obtained from $\mathrm{R}$ peaks. The mean differences \pm confidence interval were $0.16 \pm 0.18,-0.04 \pm 0.46,1.02 \pm 0.70,1.22 \pm 0.61$, and $2.31 \pm 1.09$ for increasing levels of negative pressure.

The accuracy and consistency of the algorithm enables the use of SCG as a standalone heart monitoring tool in healthy individuals at rest, and could serve as a basis for an eventual application in pathological cases.
\end{abstract}

Submitted to: Physiol. Meas. 
Accurate and consistent automatic seismocardiogram annotation without concurrent ECG2

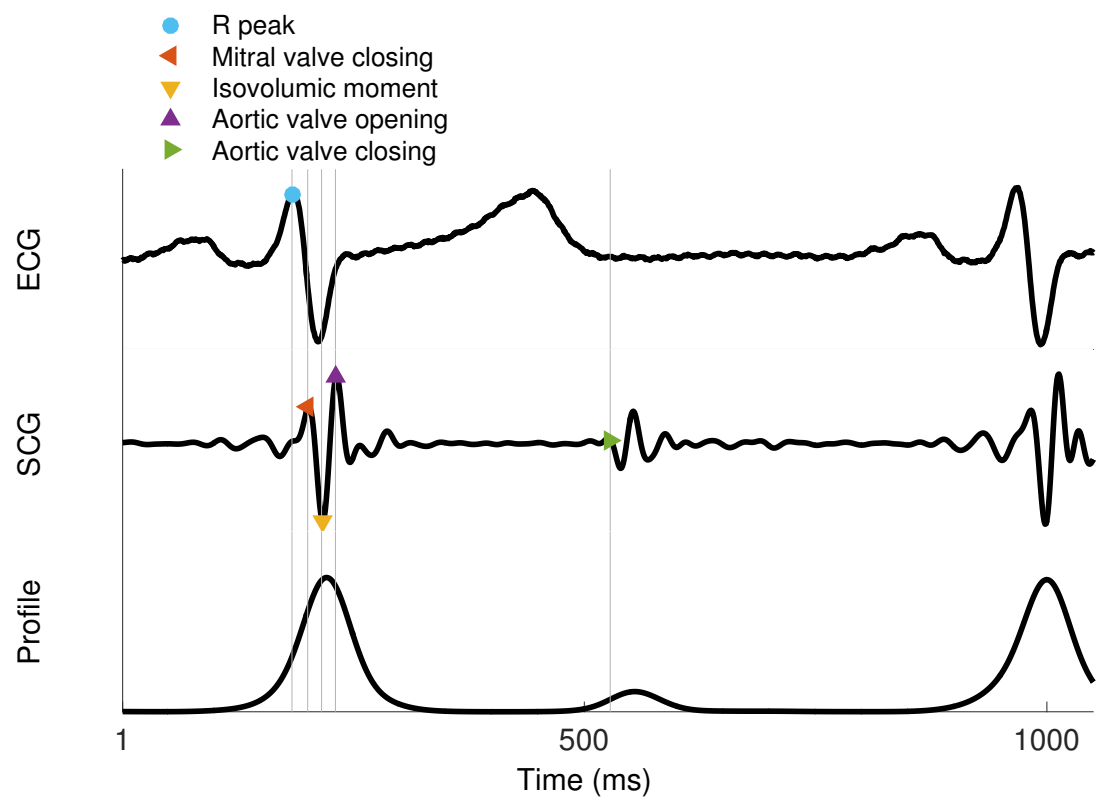

Figure 1: Example electrocardiogram (ECG), seismocardiogram (SCG), and SCG profile with relevant fiducial points. The profile is obtained from the Morlet wavelet transform of the SCG by isolating the frequency with highest power in the [20,60] $\mathrm{Hz}$ band, and returning its power in the time domain.

\section{Introduction}

Seismocardiography (SCG) is the measurement of thoracic vibrations recorded from accelerometers placed on the sternum. The signal was originally recorded by Roman Baevsky and used to monitor cosmonauts [1]. During the 90's Salerno and Zanetti used the technology for diagnosis of coronary artery disease [2]. Recent developments in MEMS accelerometer technology have rekindled research interest in the technique [3, 4].

Peaks observed in SCG have been related to significant cardiac events, the main ones being mitral valve closing (MC), aortic valve opening (AO) and isovolumic moment (IM) during the systolic cycle, and aortic valve closure (AC) during the diastolic cycle (Fig. 1). The assignment of these fiducial points was based on concurrent echocardiogram analysis with SCG morphology [5, 6]. Precise cardiac mechanical timings are obtained from SCG at less cost and lower technical difficulty than other standard techniques, most notably echocardiography and MRI. However, the measurements of these timings from SCG depend critically on accurate identification of fiducial points [7, 8].

Heart rate variability (HRV) analysis is a practical and widely used noninvasive technique to study the autonomic control of the cardiovascular system [9]. The sympathetic and parasympathetic nervous systems have complementary excitatory and inhibitory effects on the heart, respectively. The interplay between these systems cause the heart rate to exhibit regular oscillations under normal conditions. These oscillations in the steady-state heart rate are dominated by two frequencies. In the low-frequency range of $[0.04,0.15] \mathrm{Hz}$, baroreflex mediated oscillations are centered around a frequency 
of $\approx 0.1 \mathrm{~Hz}$. These oscillations are largely due to time delays inherent to the processes involved in arterial constriction and dilation [10]. Because of this, power in this band is often used to quantify sympathetic activity [11, 12]. Likewise, oscillations in the highfrequency range of $[0.15,0.4] \mathrm{Hz}$ centered around the breathing frequency are mediated by respiratory sinus arrhythmia, and are often used to quantify vagal parasympathetic activity [13].

The heartbeat intervals (HBi) used for HRV analysis are predominantly obtained from electrocardiogram (ECG) R peaks, with some exceptions such as photoplethysmography (PPG) peaks [14]. Recently, mechanical cardiac signals have provided alternatives for HBi acquisition. SCG and Ballistocardiograms (BCG) are the primary examples of such signals. BCG measures the recoil of the body due to aortic blood circulation and can be recorded from weight scales and bed sensors [4]. The comparison of HRV indices obtained from alternative and conventional methods has been used to test the consistency of the HBi estimation.

The modern-day ubiquity of accelerometers in wearable devices and smart phones could make SCG an inexpensive data acquisition tool. Such devices have been used to obtain HBi $[15,16,17]$, and the possibility of using SCG to obtain HRV indices has been reported $[17,18,19]$. BCG has also been used to consistently measure heartbeat intervals [20, 21, 22]. Additionally, correctly annotated BCG has been shown to return valid HBi and HRV indices when compared to those obtained from ECG [23, 24] and PPG [25]. In all these studies, however, the identification algorithms either depended on concurrent $\mathrm{R}$ peak identification, or did not report the accuracy of fiducial point identification. The eventual use of SCG as a stand-alone application without concurrent ECG or PPG, either at home or in the laboratory, depends on the accurate and consistent automatic identification of fiducial points with minimal user input.

The goal of this study was to develop and test SCG fiducial point identification software capable of returning valid HRV indices while requiring no input from the user. Core concepts for the algorithm were the elaboration of a function model for systolic vibration cycles, as well as an optimization function capable of accurately fitting this model to the in-vivo signal.

Accuracy of fiducial point identification differs importantly from the consistency necessary to obtain valid HRV indices. While HRV depends on consistent beat-to-beat identification of any one feature in systolic cycles, precise mechanical timings depend on the accurate identification of a particular fiducial point. To this end, our team has been involved in the development of an algorithm capable of estimating the location of IM points in some segments of a five minutes of recording [26]. The present study builds on these few identified points, filling the gaps between them.

In order to test the software's ability to correctly identify SCG fiducial points in a variety of conditions, it was applied on a dataset recorded from subjects who were exposed to lower body negative pressure (LBNP). The technique lowers blood pressure, which decreases parasympathetic and increases sympathetic outflow, which in turn increases heart rate, cardiac contractility, and total peripheral resistance. It is 
used to investigate the reaction of the cardiovascular system to stress similar to standing, hypovolemia or hemorrhage, but with minimal muscle pump effect and no vestibular stimuli, thus isolating a purer cardiovascular response [7, 27, 28]. Previous work on this dataset showed that HRV indices could be obtained from manually identified fiducial points [18].

\section{Methods}

\subsection{Data collection and annotation}

A total of 18 participants took part in this study, including three female and fifteen males, (age: $27.6 \pm 1.7$ years, weight: $71.2 \pm 5.4 \mathrm{~kg}$ and height: $174.3 \pm 3.2 \mathrm{~cm}$ ). None of the participants had any documented cardiac abnormality. Signals were recorded at the Aerospace Physiology Laboratory under an ethics approval from the Simon Fraser University Research Ethics Board. Participants followed the informed consent procedure and signed consent forms.

Each participant's lower body was placed in a negative pressure chamber and sealed at the iliac crest. Vacuum was applied to the chamber to drop the box pressure to - 20, $-30,-40$ and $-50 \mathrm{mmHg}$ progressively. The participants were kept at each stage for five minutes and were returned to normal pressure at the end of the $-50 \mathrm{mmHg}$ stage. If a participant exhibited a sudden decrease in heart rate or blood pressure or if they expressed any discomfort and wanted to stop, the negative pressure was immediately terminated.

SCG was acquired by a high sensitivity accelerometer (Brüel Kjær model 4381, Nærum, Denmark) placed on the xiphoid process of the sternum. The participants were in the supine position and the signals were recorded in back-to-front direction, perpendicular to the body surface. The ECG signal was simultaneously acquired through the NI 9205 analog input module (National Instruments, Austin, TX).

The filter banks algorithm [29] was used to identify the QRS complexes of the ECG. On the SCG, IM and AO points were assigned as the local minimum and maximum following each $\mathrm{R}$ peak. The AC point was assigned as at the beginning of the downslope of the local minimum in a window of radius $50 \mathrm{~ms}$ centred $350 \mathrm{~ms}$ after the Q-wave. The software annotations were imported to the HFM Waveform Analysis software (Heart Force Medical, Vancouver, Canada) and were manually corrected. An algorithm described in detail later in this paper (subsection 2.2) was then used to identify IM points without the use of $\mathrm{R}$ peaks.

It has been previously shown that HBi obtained with either AO peaks or IM peaks can be used to compute valid HRV indices [18]. Since previous results indicated that IM identification could be more consistent than AO identification [30], our algorithm first identified IM peaks, and from them obtained $\mathrm{AO}$ and $\mathrm{MC}$.

HBi obtained from R peaks and SCG fiducial points were computed and resampled at $5 \mathrm{~Hz}$ using a shape-preserving piecewise cubic interpolation method. We also obtained 
HBi from the identified profile peaks and resampled it using the same technique.

For all LBNP levels and participants, the power spectral density of the filtered HBi time series was computed using Welch's method [31], and normalized frequencydomain HRV indices were obtained. Normalized HRV indices measure ratios between the energy of the signal in relevant frequency bands. For example, the HF norm index is the proportion of signal's power that lies in the high-frequency spectrum $([0.15,0.4]$ $\mathrm{Hz})$

$$
\text { HF norm }=\int_{0.15}^{0.4}|\hat{s}(f)|^{2} / \int_{0.04}^{0.4}|\hat{s}(f)|^{2},
$$

where $\hat{s}$ is the Fourier transform of the signal $s$. LF norm is its low-frequency $([0.04,0.15]$ $\mathrm{Hz}$ ) analog. Frequency-domain indices were chosen over time-domain indices to reflect their dominant use in research on cardiac autonomic control, and normalized indices were chosen over absolute indices since the former are unreliable for comparisons between individuals [9].

To quantify the accuracy of the algorithm, the time difference between handannotated and automatically annotated IM, AO, and MC points was computed. For each point obtained by hand, the closest analogous point obtained automatically was identified. To prevent comparisons in heart cycles where noise prevented automatic identification of fiducial points, or where ECG noise prevented identification of the R peak, the minimum heart period using IM points obtained by hand was computed. Only the pairs of IM points that differed in time by less than $2 / 3$ the minimum HBi were then kept.

Signal analysis was performed with Matlab 2014b (Mathworks, MA, USA), and statistical analysis with JMP 11.2 (SAS Institute Inc, NC, USA). Values reported are mean $\pm 95 \%$ confidence interval. Confidence interval was computed as 1.95 times the standard error within the LBNP level.

\subsection{Detailed identification algorithm}

1. Heart-rate envelope. A moving average of the absolute value of the SCG signal was computed with parameters designed to obtain an envelope with a period of exactly one cardiac cycle [26]. The purpose of this initial envelope was to replace the ECG R-wave for cardiac cycle identification. To allow the modification of the envelope's parameters over the duration of data collection, they were optimised on ten-second segments. These parameters were the width $W_{n}$ of the window in ms, which ranged from 256 to 384, and the cutoff frequency $F_{c}$ of the lowpass filter in $\mathrm{Hz}$, which ranged from 10 to 20. The initial estimate for IM was then set to be $60 \mathrm{~ms}$ after each heartrate envelope peak. The obtained annotations were separated by gaps of at least two seconds to avoid overlap. Other gaps occurred in the pre-annotations when the standard deviation of the heart rate for a segment was above 5 bmp, usually because too much noise was present. 
The rest of the algorithm described below was developed to fill in these gaps and refine the overall estimation (Fig. 2).

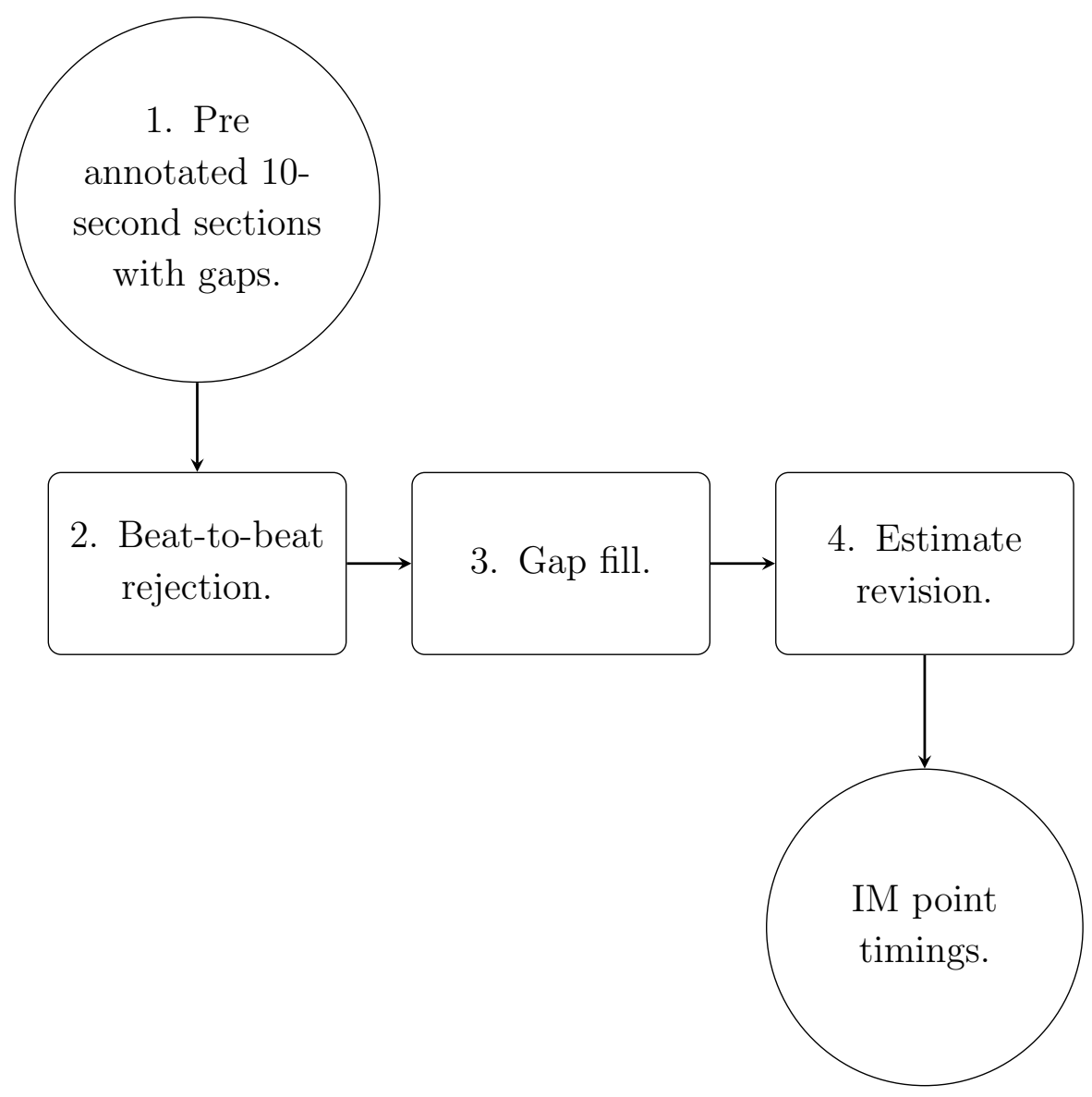

Figure 2: Summary of the isovolmic moment (IM) point identification algorithm. The input are a few very good estimates of IM points that come from a previously described algorithm [26]. The rejection step cleans the initial estimates by imposing further rigorous criteria. The next step fills the gaps between these few very good estimates. The last step uses the estimates, which are close to IM points, to determine the exact location of the IM points.

\section{Beat-to-beat rejection.}

(a) Computation of the SCG profile. This process created a time series used to identify systolic and diastolic vibrations.

A Morlet wavelet transform of order six was performed on the filtered SCG signal. This wavelet consists of a cosine wave modulated by a Gaussian function such that six periods fit in $95 \%$ of the Gaussian's area. The sum of the resulting time-frequency power over time was then obtained,

$$
S(f)=\sum_{t}|\tilde{s}(t, f)|
$$

where $\tilde{s}(t, f)$ is the wavelet transform of the signals. The frequency $f_{p}$ where $S$ 
Accurate and consistent automatic seismocardiogram annotation without concurrent ECG7

attained its maximum was computed such that

$$
S\left(f_{p}\right)=\max (S(f)) .
$$

The profile $P$ (see Figs. 1, 3, and 4 for examples) was then computed as the mean power for the frequencies around $f_{p}$,

$$
P(t)=\sum_{f_{p-2}}^{f_{p+2}} \tilde{s}(t, f) / 5 .
$$

(b) Rejection of estimates using HBi. The gap filling portions of the algorithm (step 2) relied critically on a certain number of accurate initial guesses. Because of this, strict rules were applied to first reject annotations which were doubtful. Both this and the following sub-step created more gaps in the indices obtained from the pre-annotations, and widened the ones that already existed.

HBi was computed for each pre-annotated section. Within these sections, if successive intervals differed by more than a threshold value $T$, they were rejected. If 3 or more consecutive heart beats did not deviate from each other by more than $T$, they were kept.

This process assumed that heart period rarely changes by more than some value in a single beat. Here, $T$ was set as 3 times the interquartile range of HBi including all sections if this value was $\in[120,300] \mathrm{ms}$. Otherwise, it was set at either 120 or $300 \mathrm{~ms}$, whichever was closest. The threshold $T$ was purposely low to favour false negatives over false positives.

$$
T= \begin{cases}3 \cdot i q r(\mathrm{HBi}) & \text { if } 3 \cdot i q r(\mathrm{HBi}) \in[120,300] \\ 300 & \text { if } 3 \cdot i q r(\mathrm{HBi})>300 \\ 120 & \text { if } 3 \cdot i q r(\mathrm{HBi})<120,\end{cases}
$$

where $i q r$ is the interquartile range. The interquartile range was chosen for its ability to ignore outliers.

(c) Rejection of estimates that look like diastolic cycles. The misidentification of diastolic cycles as systolic cycles for entire sections was a recurrent problem. Because such systematic misidentifications did not affect $\mathrm{HBi}$, and thus were not picked up by $T$, another rejection criterion had to be designed.

For each identified profile peak $p_{n}$ in the section, the profile peaks directly before $\left(p_{n-1}\right)$ and after it $\left(p_{n+1}\right)$ were identified. For the section, if $p_{n}-p_{n-1}<p_{n+1}-p_{n}$ for all initially identified peaks, the section was rejected (Fig. 3). This process assumed that systolic cycles were closer to the subsequent diastolic cycle than to the preceding diastolic cycle.

This process was repeated after each other step.

3. Gap fill. There were three kinds of gaps: 2-second gaps to avoid overlaps in the initial guesses, 10-second gaps because pre-annotation failed for an entire section (usually because of noise), and gaps that were created by the previous step (2).

The gap filling process relied on minimizing the standard deviation of the HBi time series, taking into account the sections immediately preceding and following the gap. 
Accurate and consistent automatic seismocardiogram annotation without concurrent ECG8

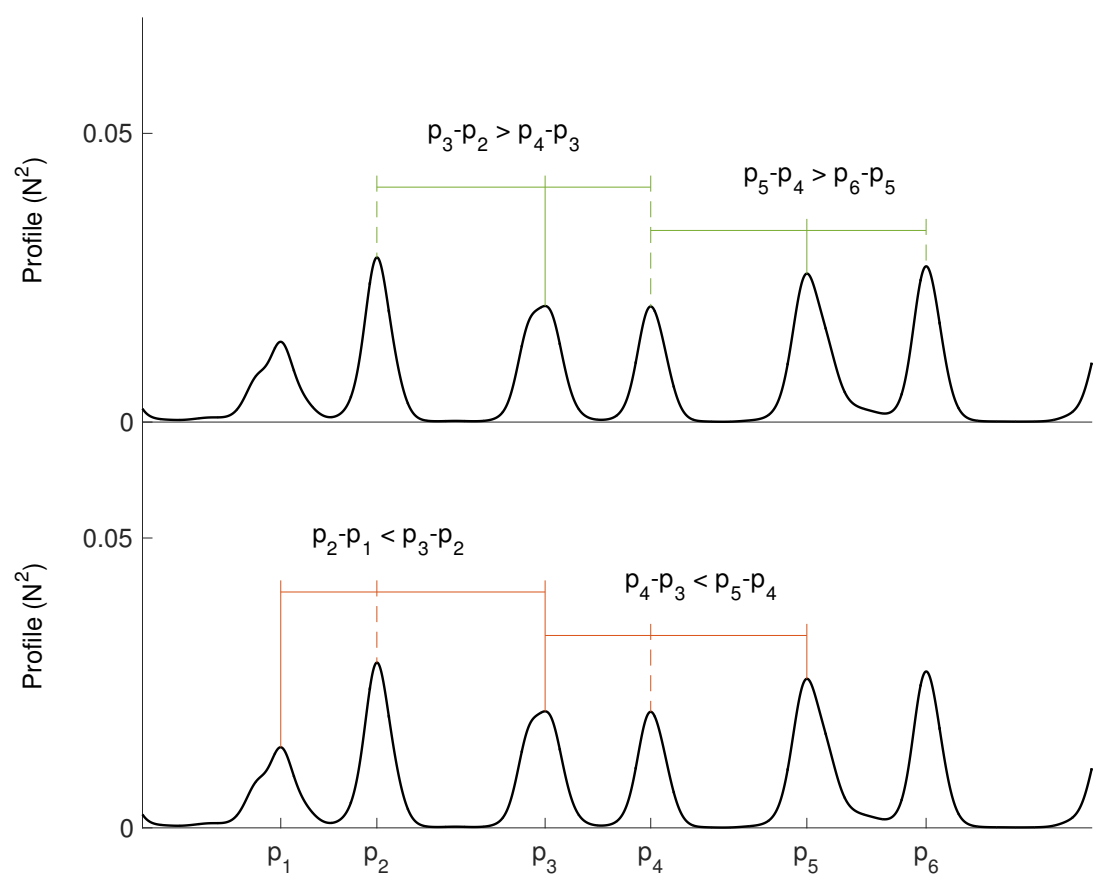

Figure 3: Example SCG profile showing exclusion criterion for diastolic peaks labeled mistakenly as systolic peaks. Here, odd peaks $\left\{p_{1}, p_{3}, p_{5}\right\}$ are systolic, and even peaks $\left\{p_{2}, p_{4}, p_{6}\right\}$ are diastolic. Top Correctly identified systolic peaks are closer to the next diastolic peak than the previous diastolic peak. Bottom. Incorrectly identified diastolic peaks are closer to the previous systolic peak than the next systolic peak.

For a given gap, candidate profile peaks were identified as local maxima higher than half the median of the relevant sections (Fig. 4). The sections immediately preceding and following the gap were appended with all possible combinations of the candidates. For each combination, standard deviation of HBi was computed. The set of candidates that minimized the standard deviation were kept for further analysis. This process relied on the assumption that fluctuations in HBi over a limited time are small.

If a gap between sections was longer than $10 \mathrm{~s}$, the process proceeded analogously to the manner previously described, but included only the section immediately preceding the gap and $3 \mathrm{~s}$ of the beginning of the gap profile. This initial narrowing of the gap was followed by the same technique, but including the section immediately following the gap and $3 \mathrm{~s}$ of the ending of the gap profile. This gap-narrowing process was repeated until the gap became shorter than $10 \mathrm{~s}$.

The output of this step, a sequence of systolic profile peaks, was also used to compute an HBi time series.

4. Estimate revision.

(a) Fit of a model to the participant's median systolic cycle. This process assumed that at least half of the profile peaks correctly identified systolic cycles, and that the absolute minimum of the SCG during systolic cycles is consistent in time. 
Accurate and consistent automatic seismocardiogram annotation without concurrent ECG9

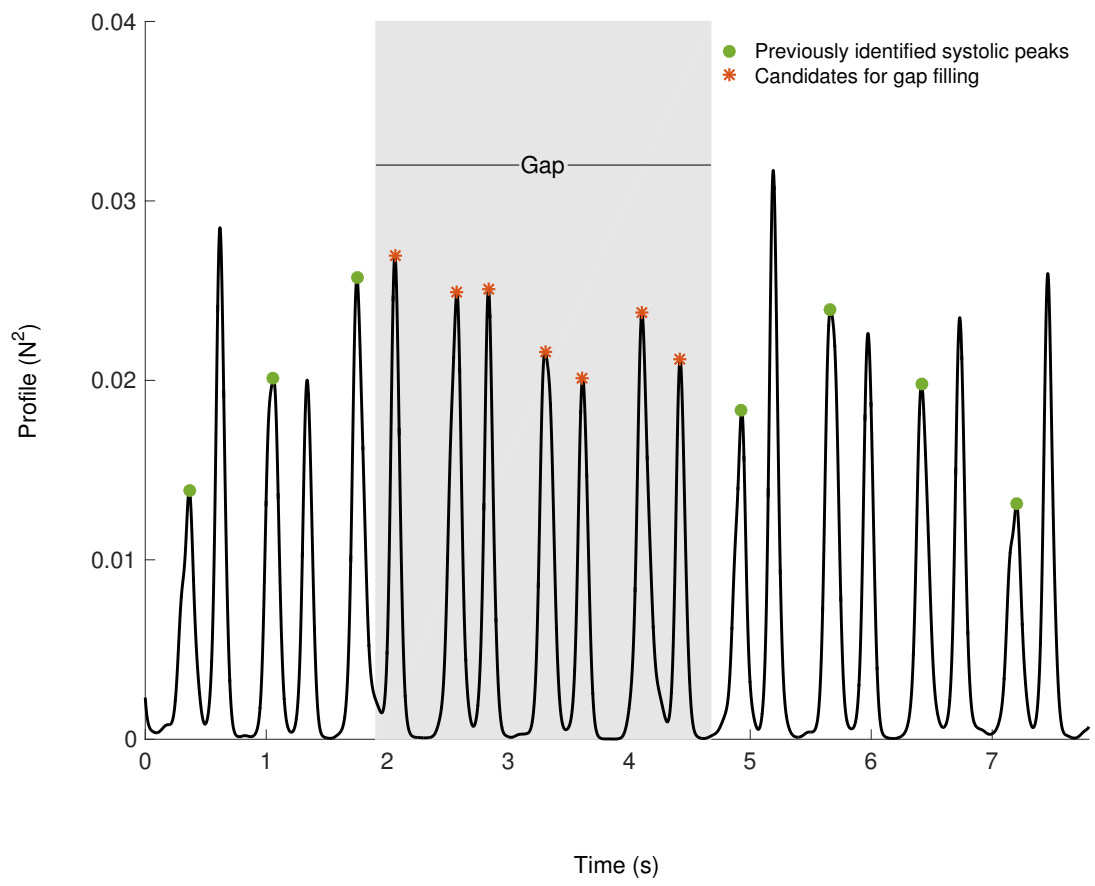

Figure 4: Example SCG profile with previously identified systolic peaks in green circles and gap-filling candidates in red asterisks. To identify which candidates were correct, the heart period time series was computed using the previously identified systolic peaks and all possible candidate combinations. The correct candidates were then identified as the combination that minimized the standard deviation of the heart period.

For each profile peak, the absolute minimum of the SCG signal within a 200 ms radius was identified. Segments of $400 \mathrm{~ms}$ of the SCG signal were then centered around those minima, and their median was computed (Fig. 5 Top). The resulting median was normalized by dividing it by its maximum absolute value. The normalized median was windowed by the function $\exp \left(-(t / 100)^{2}\right)$ to emphasize vibrational peaks that occurred relatively close to the segment's minimum. The value of 100 for the time constant of the window was not critical, and values from 70 to 150 yielded similar results.

The model was defined as the function

$$
f\left(t, x_{0}, p, \phi_{i}\right)=A \cdot \sin \left(\left(t-x_{0}\right) \cdot \frac{2 \pi}{p}\right) \cdot \sum_{i=1}^{5} \phi_{i}
$$

where $A$ normalized the maximum to $1, t$ was time, $x_{0}$ was a time shift which was constrained to $[-30,30] \mathrm{ms}$ and represented the point exactly between IM and AO, $p$ was the period which was constrained to $[20,60] \mathrm{ms}$, and each $\phi_{i}$ was a compactly supported gaussian defined as

$$
\phi_{i}=\left\{\begin{array}{lc}
B \cdot \exp \left[\left(\frac{-2\left(x-x_{i}\right)}{p}\right)^{2}\right], & \text { for }\left|x-x_{i}\right|<p / 2 \\
0, & \text { otherwise }
\end{array}\right.
$$

where the $x_{i}$ were centered at the $\mathrm{MC}$, IM, AO, post-AO, and post-post- 
AO peaks, respectively (Fig. 5). The amplitudes $B$ of the gaussians were constrained, respectively from left to right, to $[0.1,0.6],[0.3,1],[0.9,1],[0.1,1]$, and $[0.1,1]$. The amplitude constraints were designed from repeated observation to emulate normal signal morphology. The function in Equation 7 was designed for this study to match repeated empirical observations on normal SCG cycles, with the assumption that the frequency of SCG fiducial points was regular within cycles. The model was inspired by the Morlet wavelet, replacing its single modulating Gaussian with five narrower ones.

To determine the initial values of the parameters, the two SCG segment minima, and two SCG segment maxima that differed by at least $20 \mathrm{~ms}$ were identified. By comparing the timings of these extrema it was determined if the left-most maximum was a $\mathrm{MC}$ point or an $\mathrm{AO}$ point. The initial period $p$ was then set to be the distance between these two maxima, and either the MC and AO amplitudes, or the AO and post-post-AO amplitudes to their respective SCG segment maxima values. The initial IM and post-AO amplitudes were set to their respective SCG segment minima values. If the two maxima were not both above 0.6 , the initial values were set by default to $p=40$, and amplitudes $=\{0.2,0.8,0.9,0.5,0.1\}$.

The model was fitted by a simplex search method [32], implemented in Matlab as fminsearch, to minimize a distance function $D$ defined as

$$
D=\frac{d_{1}}{p} \cdot \sum_{t} w(t) \cdot d_{2}(t)
$$

where $d_{1}$ is the mean distance between the two maxima and two minima of the model and signal, respectively, $d_{2}$ is the square point-wise difference between the signal and the model, and $w(t)$ is a weighing function that favours the beginning of the cycle over its end. The weighing function $w(t)$ was necessary because, while inter-participant differences in the beginning of systolic cycles are minimal, differences in their end are substantial. The weighing function $w(t)$ was then designed as a decaying sigmoid defined as

$$
w(t)=0.8 \cdot \tan ^{-1}(-0.05 \cdot(t-(3.5 \cdot p)))+1.2 .
$$

The parameters of the sigmoid were chosen for $w(t)$ to be $\approx 1$ before $\mathrm{AO}$, and drop to $\approx 0$ beyond post-post-AO. These parameters were optimized through trial and error, and variations of the order of $\pm 20 \%$ affected the results only marginally.

The model resulting from this process was assumed to represent a generic systolic cycle for the participant at that LBNP level.

(b) Fit of a model to each systolic cycle. This process fitted the generic model obtained in step 4(a) to all systolic cycles of the participant, for a particular level of LBNP. For each identified profile peak, the distance function $D$ (Equation 8) was minimized with respects to $x_{0}$ only (Equation 7 ).

This process implicitly returned timing estimates for MC, IM, and AO points. 


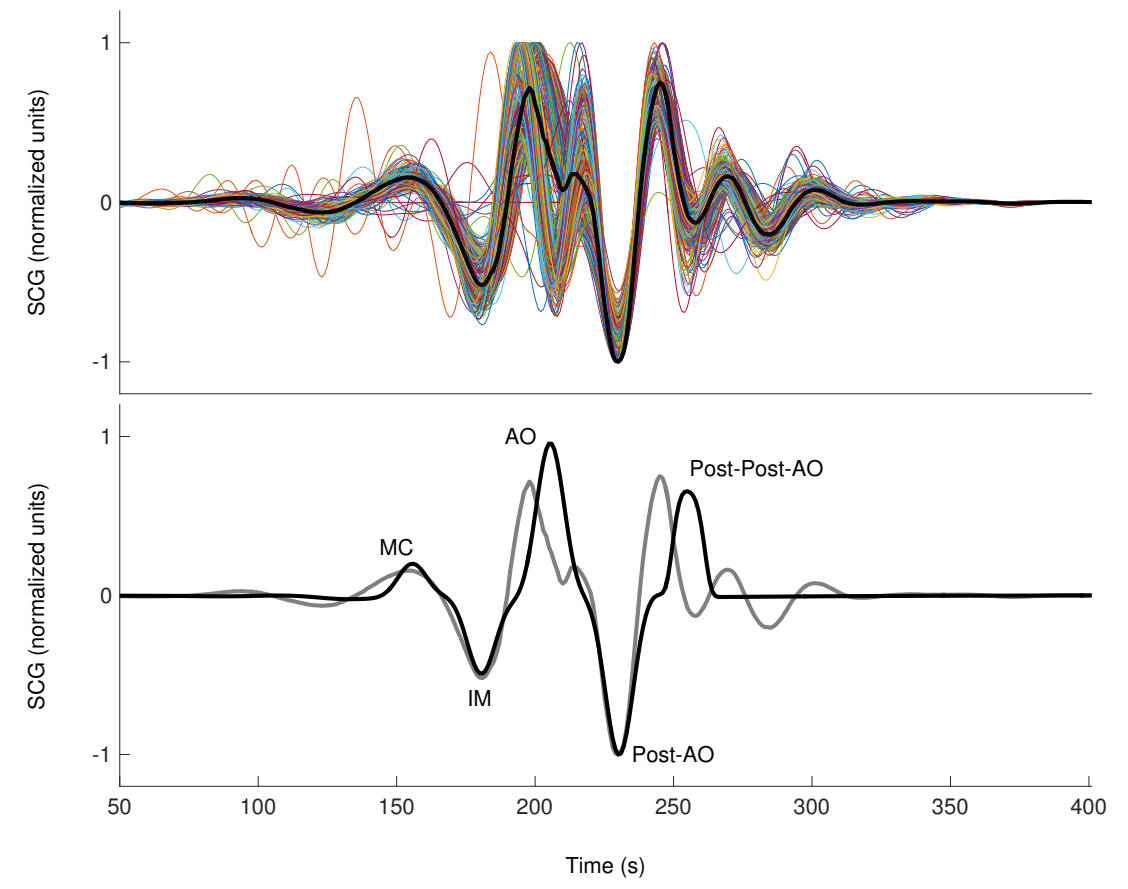

Figure 5: Top Seismocardiogram within $200 \mathrm{~ms}$ of all identified profile peaks $(n=349)$ of a single participant, centered around their minimums. The median of the resulting signals is shown (black). All segments were normalized to their maximum absolute value. Identifying fiducial points to local maxima or minima is a standard technique for automatic identification $[15,16,17,19]$ and for this participant would return at least some misidentified peaks. Bottom The median SCG obtained above (grey) with its corresponding model (black). The model is the function in equation 7 with $x_{0}=-8$ $\mathrm{ms}, p=50 \mathrm{~ms}$, and the amplitudes of the gaussians $=\{0.2,0.5,0.9,1,0.8\}$, respectively. The model returns implicit timing estimates for the SCG fiducial points.

(c) Refinement of each estimate. While SCG fiducial points are always local signal extrema, the timings estimates obtained in the previous sub-step were often a slight distance from the nearest relevant extremum. For each estimate, the SCG extremum within a window of radius $p / 4$ was identified. The estimate was then moved to the identified extremum and these steps were repeated until a minimum relative to the window was reached.

\section{Results}

The measurement of accuracy compared automatic annotations to hand annotations. For each LBNP level and each subject, the percentage of accurately identified IM points was computed. An identification was considered accurate when the difference between the timing of the hand-identified IM point and the automatically identified IM point was zero. Table 1 shows the mean percentage of accurately annotated cycles with respect to the total number of cycles where both automatic- and hand-annotations were present 
Table 1: Mean \pm confidence interval in percentage (\%) of accuracy, missing annotations, and isovolumic moment heart rate variability index errors for all participants $(n=18)$ and each level of $L B N P$.

\begin{tabular}{lccccc}
\hline & Baseline & -20 & -30 & -40 & -50 \\
\hline IM accuracy & $97.2 \pm 3.7$ & $93.0 \pm 4.6$ & $76.9 \pm 14.9$ & $61.6 \pm 17.2$ & $65.0 \pm 14.0$ \\
AO accuracy & $96.2 \pm 4.2$ & $91.7 \pm 5.5$ & $73.8 \pm 16.0$ & $58.8 \pm 19.1$ & $60.4 \pm 17.4$ \\
MC accuracy & $96.2 \pm 3.7$ & $93.5 \pm 4.3$ & $80.9 \pm 14.1$ & $70.7 \pm 15.4$ & $72.8 \pm 12.7$ \\
& & & & & \\
Missing IM & $9 \pm 4.5$ & $16.5 \pm 10.1$ & $19.6 \pm 10.7$ & $26.1 \pm 9.2$ & $22.8 \pm 6.5$ \\
$\begin{array}{l}\text { (automatic) } \\
\text { Missing IM }\end{array}$ & $12 \pm 6.7$ & $12.5 \pm 7.3$ & $13.5 \pm 7.0$ & $13.2 \pm 7.0$ & $12.0 \pm 5.6$ \\
$\begin{array}{l}\text { (hand) } \\
\text { Missing R waves }\end{array}$ & $0.5 \pm 0.9$ & $0.3 \pm 0.3$ & $1.0 \pm 1.9$ & $0.3 \pm 0.4$ & $0.2 \pm 0.3$ \\
& & & & & \\
HF norm err. & $-1.3 \pm 1.7$ & $-0.2 \pm 2.9$ & $-5.3 \pm 3.6^{*}$ & $-6.3 \pm 3.2^{*}$ & $-9.5 \pm 4.6^{*}$ \\
LF norm err. & $1.3 \pm 1.9$ & $0.2 \pm 3.3$ & $5.7 \pm 3.7^{*}$ & $6.3 \pm 3.7^{*}$ & $10.0 \pm 4.8^{*}$ \\
LF/HF err. & $0.16 \pm 0.18$ & $0.04 \pm 0.46$ & $1.02 \pm 0.70^{*}$ & $1.22 \pm 0.61^{*}$ & $2.30 \pm 1.09^{*}$ \\
\hline
\end{tabular}

The accuracy of isovolumic moment (IM), aortic valve opening (AO), and mitral valve closing (MC) are the percentage of total cardiac cycles where both automatic- and handannotations were present that were correctly annotated. Missing annotations are the percentage of cardiac cycles where a particular annotation was absent. High frequency, low-frequency normalized power, and their ratio (HF norm, LF norm, and LF/HF) values are the differences between indices obtained from $\mathrm{R}$ waves and those obtained from automatic IM annotation. For HRV index errors, values statistically different from zero are indicated with $\mathrm{a}^{*}(p<0.05)$.

for all participants and each level of LBNP.

The accuracy computations compared annotations located in the same cardiac cycle. Because of noise or other factors, some cycles lacked either a hand-annotated IM point, an automatically annotated IM point, or an $\mathrm{R}$ peak. Table 1 shows the mean percentage of the total cardiac cycles for all participants that were missing either of these three annotations.

The quality of the information that can be obtained from automatically annotated SCG was quantified by comparing normalized HRV indices obtained from the gold standard RR intervals to those obtained with IM points (Table 1). At levels of LBNP more intense than $-30 \mathrm{mmHg}$, all three indices obtained automatically were statistically different than those obtained from RR intervals. Identified systolic profile peaks were 
Accurate and consistent automatic seismocardiogram annotation without concurrent ECG13
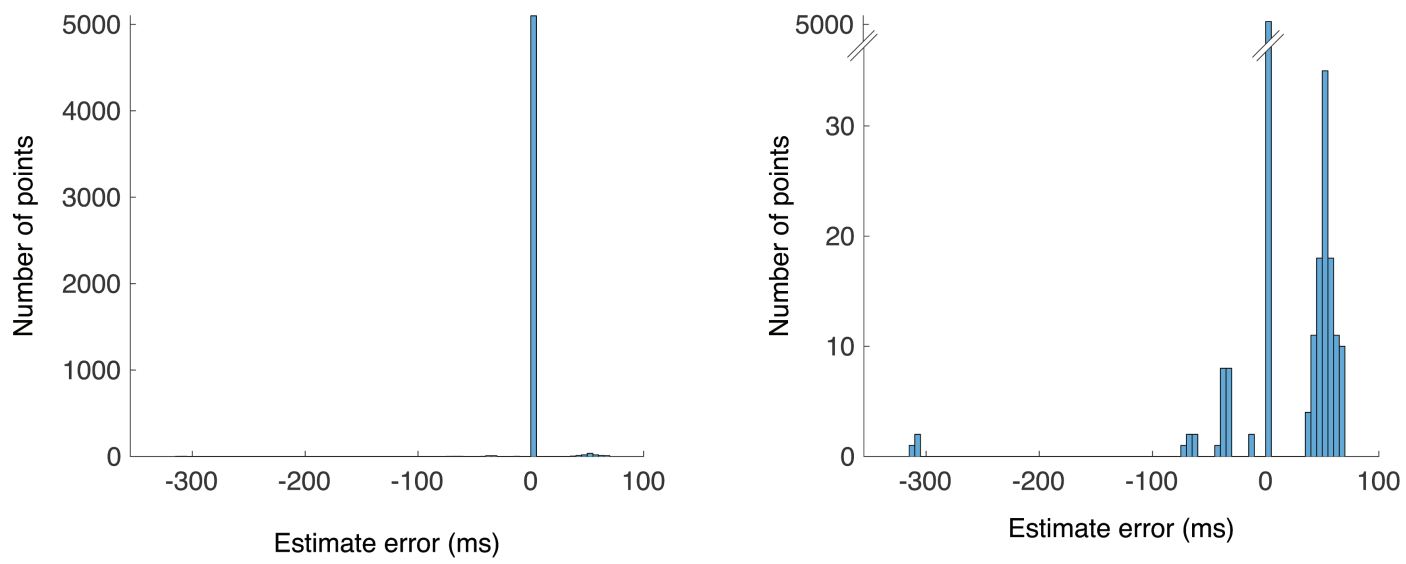

Figure 6: Histogram of the estimation errors of the algorithm at baseline for all participants $(n=18)$. The mean error is $0.72 \pm 0.30 \mathrm{~ms}$. Right A truncated version of the histogram on the left highlighting the inaccuracies. The groups of errors centered at $\approx \pm 50 \mathrm{~ms}$ represent errors wherein either the previous or subsequent SCG peaks were misidentified as the isovolumic moments. The few errors near $-300 \mathrm{~ms}$ represent diastolic cycles mistaken for systolic cycles.

also used to compute normalized HRV indices and were compared to those obtained from RR intervals (Table 2).

The root-mean-square error (RMSE) for IM identification was 8.4, 34.3, 28.2, 70.3, and $72.9 \mathrm{~ms}$ for increasing levels of LBNP. For MC identification, RMSE was 9.6, 33.6, 27.0, 70.0, and 71.1 ms, respectively. For AO identification, it was 7.5, 33.5, 29.2, 71.6, and $73.6 \mathrm{~ms}$, respectively.

The RMSE for heart beat interval for IM-IM versus $\mathrm{RR}$ was 40, 71, 26, 51, and $27 \mathrm{~ms}$ for increasing levels of LBNP. For those obtained from profile peaks versus RR intervals, RMSE was 40, 71, 25, 44, ans 28 ms, respectively.

Table 2: Mean \pm confidence interval in percentage (\%) of systolic profile peak heart rate variability index errors for all participants $(n=18)$ and each level of LBNP.

\begin{tabular}{lccccc}
\hline & Baseline & -20 & -30 & -40 & -50 \\
\hline HF norm err. & $-1.9 \pm 1.4^{*}$ & $-0.7 \pm 4^{*}$ & $-3.6 \pm 2.7^{*}$ & $-5.2 \pm 3.7^{*}$ & $-6.6 \pm 3.3^{*}$ \\
LF norm err. & $1.9 \pm 1.5^{*}$ & $0.6 \pm 4.2^{*}$ & $4 \pm 3.2^{*}$ & $5.4 \pm 3.7^{*}$ & $7.3 \pm 3.5^{*}$ \\
LF/HF err. & $0.24 \pm 0.22^{*}$ & $0.12 \pm 0.59$ & $0.77 \pm 0.59^{*}$ & $1.07 \pm 0.69^{*}$ & $1.93 \pm 1.12^{*}$
\end{tabular}

High frequency, low-frequency normalized power, and their ratio (HF norm, LF norm, and $\mathrm{LF} / \mathrm{HF}$ ) values are the differences between indices obtained from $\mathrm{R}$ waves and those obtained from profile peaks. Values statistically different from zero are indicated with $\mathrm{a}^{*}(p<0.05)$. 
Accurate and consistent automatic seismocardiogram annotation without concurrent ECG14

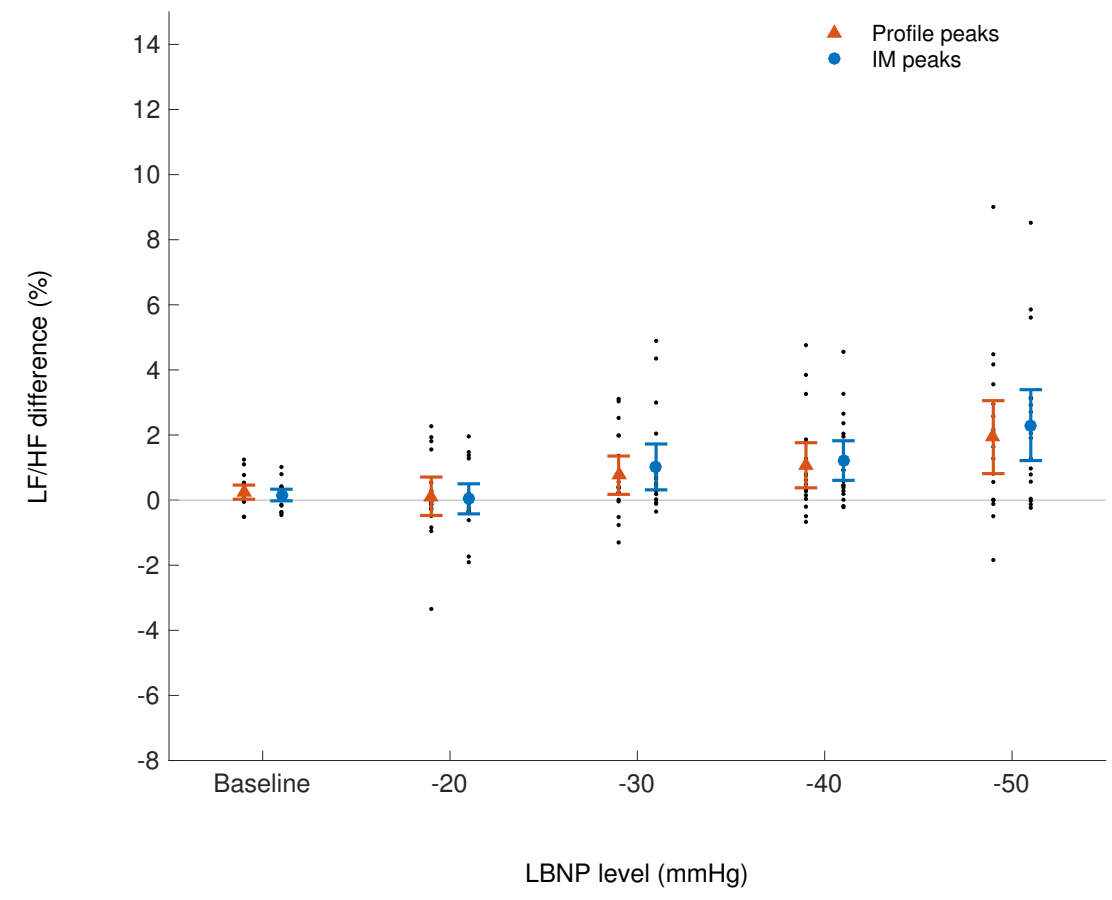

Figure 7: Mean \pm confidence interval of low-frequency to high-frequency power ratio ( $\mathrm{LF} / \mathrm{HF}$ ) differences from heartbeat interval obtained from $\mathrm{R}$ peaks for all participants $(n=18)$ and each level of LBNP. Orange triangles show values for systolic profile peaks, and blue circles show values for isovolumic moments.

\section{Discussion}

A new algorithm for the identification of the IM fiducial point on SCG without the use of ECG was tested in its accuracy and consistency across levels of LBNP.

The methodology described in this study uses new techniques which distinguish it from previously described algorithms; firstly by the design of a systolic vibration model, and secondly by its fit using a comprehensive distance function. The systolic vibration model was able to overcome the difficulties created by multiple extrema in the vicinity of true fiducial points (so-called bunny-eared peaks), allowed concurrent estimation of all systolic fiducial points, and returned a representation of participants' general SCG morphology. The distance function that was minimized to fit the model took into account the mean square difference, the distances between the two highest and two lowest peaks, as well as a weighing function penalizing the earlier portion of each systolic vibration cycle. This distance function successfully mitigated systematic errors caused by longer-than-average systolic vibrations and inconsistent absolute extrema by considering both simultaneously.

The accuracy of IM identification was tested by the comparison of the timings obtained automatically to timings obtained by hand. The levels of accuracy reached at baseline and $-20 \mathrm{mmHg}$ of LBNP would be largely sufficient for applications that require either the exact amplitude, frequency, or timing of the systolic SCG fiducial 
points. As such, it could provide sufficient data to track changes in cardiac function during hemorrhage [7].

The consistency of the algorithm was tested by comparing HRV indices obtained from $\mathrm{R}$ peaks to those obtained from automatically identified IM points and profile peaks. At baseline and $-20 \mathrm{mmHg}$ of LBNP, the IM-obtained indices were not statistically different than the $\mathrm{R}$ peak-obtained indices, but most importantly, they had physiologically insignificant mean differences, and relatively narrow confidence intervals. These results support the claim that in some conditions, the algorithm is consistent enough to perform HRV analysis without additional concurrent signals or timings.

The HRV index differences obtained from the profile peaks were slightly smaller than those obtained from IM points, although they were never statistically different from each other. This result illustrates the category of errors that arose from the model fitting. Because the profile identifies the systolic cycle rather than the exact location of the SCG fiducial point, the HBi obtained from profile peaks can sometimes be smoother, which may translate into better index estimates. This brings to light a trade-off that SCG identification algorithms make between accuracy and consistency, and could provide an argument for hybrid approaches where exact IM peaks would only be obtained from profile peaks in specific relevant heart cycles.

It is possible that the changes in intra-thoracic pressure and volume caused by LBNP affect the mechanical properties of the heart and other constituent tissue through which these vibrations propagate. The relationship in time between SCG fiducial points and valve openings has been shown to be affected by certain pathologies [5], and it is not unreasonable to think LBNP might affect it as well. SCG research would benefit from an in-depth analysis of the exact causes of its fiducial points.

It is interesting to note that there was consistently slightly above $10 \%$ of cardiac cycles that had automatically identified IM points that did not have hand-annotations. There were also some cycles where IM points were identified automatically where no R peak was identified. This points to one of the strength of automatically annotated SCG to support concurrent cardiac measurement, both as a fail-safe, and as a low-marginalcost way of complementing already acquired data.

\subsection{Limitations}

The algorithm became much less accurate and consistent at levels of LBNP more intense than $-20 \mathrm{mmHg}$. This presents the clearest limitation of the study as heart monitoring is most useful in abnormal situations. This loss of accuracy was not, however, caused by overfitting it to baseline data. In fact, most fitting efforts were exerted at more intense levels of LBNP, and the difficulties arose from increased inter-participant differences and noise. Figure 8 illustrates some of these inconsistent changes, for which a parameter change that would increase accuracy for one participant would decrease it for another.

Figure 9 shows an increase in the noise of an example participant's signal caused by higher levels of LBNP. Such noise could potentially be created by participants' 
Accurate and consistent automatic seismocardiogram annotation without concurrent ECG16

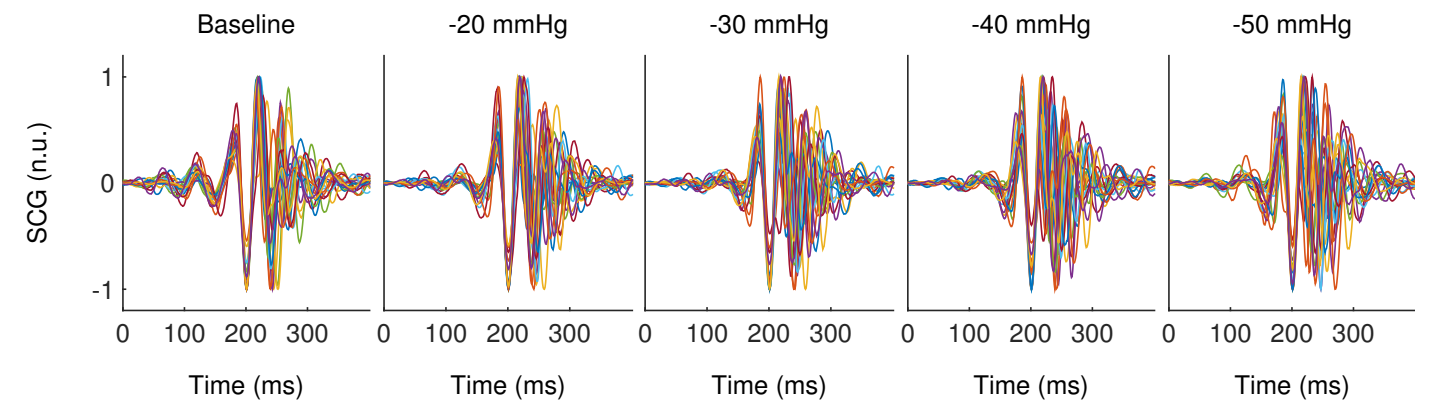

Figure 8: Median systolic cycles for all participants $(n=18)$ across all levels of LBNP. Each median cycle was centered at the hand-identified isovolumic moment fiducial point. To ease comparisons between participants, each segment was divided by its maximum absolute value.

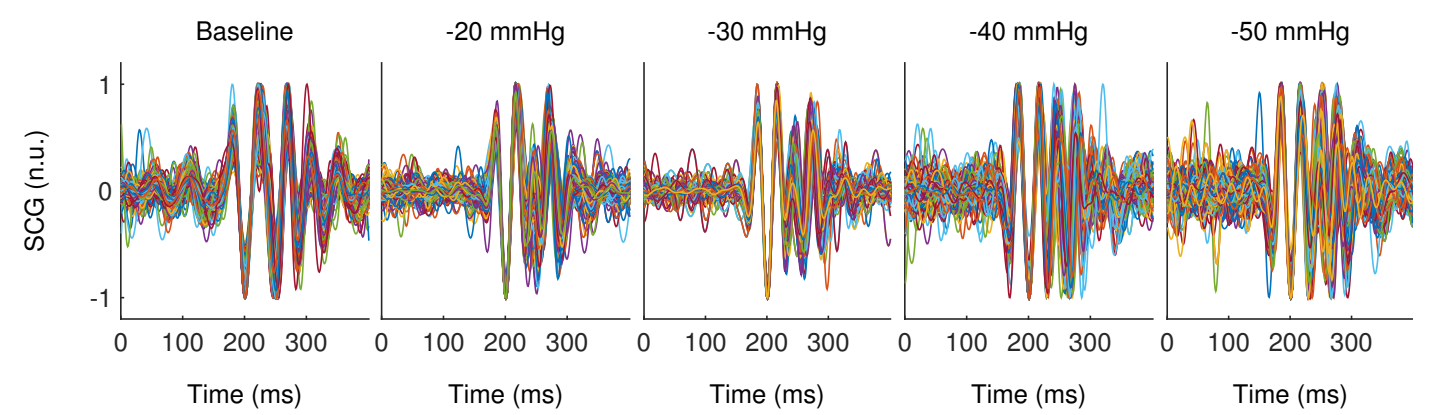

Figure 9: All systolic cycles for an example participant across all levels of LBNP. Each cycle was centered at the hand-identified isovolumic moment fiducial point. To ease comparisons between cycles, each segment was divided by its maximum absolute value.

movements or twitches, changes in the geometry and physical properties of thoracic organs due for example to volume shifts, changes in cardio-vascular dynamics due to decreased pre-load and after-load, etc. For this example participant, higher levels of LBNP also increased the number of vibration peaks with amplitudes comparable to those of fiducial points throughout the cycle. Noise and non-fiducial peaks of high amplitude are important sources of error for the algorithm. They both modify the profile, making it vary from the norm, and create errors in model fitting.

At all levels of LBNP, the inaccuracies of the annotation can be classified in two groups. In the first case the SCG peak directly next to the correct one was misidentified as IM. These errors arose from the model fit, and were consistently of $\approx \pm 50 \mathrm{~ms}$. In the second case, a diastolic vibration was misidentified as a systolic vibration. These errors arose from the gap filling algorithm, and were consistently of $\approx-300 \mathrm{~ms}$ (Fig. $6)$. The percentage of cardiac cycles in which hand-annotations are present but where automatic annotations are absent went up with increased LBNP. Although this can partly be explained by increased noise and an altered signal morphology, the algorithm itself was also responsible. Indeed, it was built in such a way that each individual identification depended on the few preceding and few subsequent identifications. As 
such, if sporadic noise prevented identification in a single cycle, it would also prevent it in surrounding cycles. This compounding-mistakes effect could account for the abruptness of the deterioration of the accuracy. In this sense, the algorithm would be either very accurate, or not accurate at all. This is an important limitation of the algorithm which would need to be addressed before its use for monitoring pathological situations.

The many parameters of the algorithm were optimized by trial and error and no systematic in-depth analysis of their cross-sensitivity was performed. Generally, it was found that a change in one parameter that generated marginal gains in accuracy for one participant or one level of LBNP usually created a analogous marginal losses in accuracy for another participant or level of LBNP. Furthermore, the robustness of the algorithm to changes in parameters was supported by the function-fitting process which was built to include a comprehensive distance function (Equation 8) and an initial fit to the median cycle (Fig. 5). A future version of the algorithm would benefit from an optimization of parameters according to more easily available initial estimates upstream in the algorithm. For example, it is possible to estimate heart rate before the model is fit. This information could be used in the rejection step 2(c), which RR intervals smaller than $\approx 650 \mathrm{~ms}$ render less reliable. In our cohort, one participant had 24 systolic cycles rejected by this sub-step although they were accurate, all of which occurred when the associated RR interval was less than $660 \mathrm{~ms}$. Two other participants also had 3 cycles rejected. Most probably, an algorithm based on this one, but incorporating minimal user input could also increase its performance. By training users to correctly identify a small number of IM points on test signals, then on their own, it would be simple to adjust the parameters of the model and weighing function to reflect individual morphologies.

\section{Conclusion}

In supine position at low levels of LBNP, the algorithm was both accurate enough to obtain precise IM timings, and consistent enough to obtain meaningful HRV indices. These results open opportunities for stand-alone applications of SCG for home use as well as in laboratories. It is clear, however, that the most valuable uses of SCG as a cardiac monitoring tool would be in abnormal cases that are neither represented by the demographics of this study's participants or in relatively quiet conditions that exist at LBNP intensities below $-30 \mathrm{mmHg}$.

Of particular contemporary relevance are potential uses of SCG for monitoring the elderly at home as changes in the cardiovascular system due to ageing would undoubtedly be reflected in SCG. Although, the characteristics of the population sample included in this study do not allow conclusions in this regard, it is conceivable that the overall amplitude of the signal would lessen due to the heart's diminishing contractility, and that the frequency content of the systolic vibration could shift to lower frequencies due to bone density loss. More directly, the work presented in this study offers a straightforward application for the monitoring of arrythmias, the prevalence of which is known to increase with age. 
Generally, because accelerometers are becoming ubiquitous and inexpensive, SCG is positioning itself to become an important part of out-of-clinic cardiac monitoring. Theoretically, any thoracic condition that alters the mechanics responsible for SCG could be identified and monitored, most obviously pericarditis and pleurisy where friction could dramatically increase some peaks while removing others, as well as cardiomyopathies and cardiac insufficiencies where amplitudes should decrease in general. Whether the modalities for this monitoring include the exact detailed annotation of a few clean beats, or overall average statistics like heart rate, the need for stand-alone annotation algorithms is clear.

The ability of this study's algorithm to annotate SCG in relatively good conditions is a step in the right direction, and solves many of the initial problems encountered in attempting to annotate SCG automatically. With some modifications, either through adaptive parameter optimization or initial user input, it could potentially be adapted to annotate abnormal cases.

\section{Acknowledgments}

This research was supported in part by an NSERC/CIHR Collaborative Health Research Project grant (201110) to AP Blaber. The data acquisition was sponsored by Heart Force Medical Inc.

\section{References}

[1] R M Baevskii, A D Egorov, and L A Kazarian. The Method of Seismocardiography. Kardiologiia, 18:87-9, January 1964.

[2] DM Salerno, JM Zanetti, LC Poliac, RS Crow, PJ Hannan, K Wang, IF Goldenberg, and RA Van Tassel. Exercise seismocardiography for detection of coronary artery disease. American journal of noninvasive cardiology, 6(5):321-330, 1992.

[3] John M Zanetti and Kouhyar Tavakolian. Seismocardiography : Past, Present and Future. In IEEE Engineering in Medicine and Biology Society Conference, pages 7004-7007, Osaka, Japan, 2013.

[4] Omer Inan, Pierre-Francois Migeotte, Kwang-Suk Park, Mozziyar Etemadi, Kouhyar Tavakolian, Ramon Casanella, John Zanetti, Jens Tank, Irina Funtova, Kim Prisk, and Marco Di Rienzo. Ballistocardiography and Seismocardiography: A Review of Recent Advances. IEEE Journal of Biomedical and Health Informatics, 19(4):1414-1427, 2014.

[5] RS Crow, P Hannan, D Jacobs, L Hedquist, and D Salerno. Relationship between seismocardiogram and echocardiogram for events in the cardiac cycle. American journal of Noninvasive Cardiology, 8(39):39-46, 1994.

[6] Kouhyar Tavakolian, Andrew P Blaber, Brandon Ngai, Bozena Kaminska, Engineering Science, British Columbia, and Biomedical Physiology. Estimation of Hemodynamic Parameters from Seismocardiogram. In Computing in Cardiology, pages 1055-1058, Belfast, 2010.

[7] Kouhyar Tavakolian, Guy A Dumont, Geoffrey Houlton, and Andrew P Blaber. Precordial vibrations provide noninvasive detection of early-stage hemorrhage. Shock, 41(2):91-96, 2014.

[8] David M Salerno and J Zanetti. Seismocardiography for monitoring changes in left ventricular function during ischemia. CHEST Journal, 100(4):991-993, 1991.

[9] Task Force of the European Society of Cardiology et al. Heart rate variability standards of measurement, physiological interpretation, and clinical use. Eur Heart J, 17:354-381, 1996. 
[10] Claude Julien. The enigma of mayer waves: facts and models. Cardiovascular research, 70(1):1221, 2006.

[11] J Philip Saul, RD Berger, P Albrecht, SP Stein, M Hui Chen, and RJt Cohen. Transfer function analysis of the circulation: unique insights into cardiovascular regulation. American Journal of Physiology-Heart and Circulatory Physiology, 261(4):H1231-H1245, 1991.

[12] Philippe Van De Borne, Mohsen Rahnama, Silvia Mezzetti, Nicola Montano, Alberto Porta, Jean Paul Degaute, and Virend K Somers. Contrasting effects of phentolamine and nitroprusside on neural and cardiovascular variability. American Journal of Physiology-Heart and Circulatory Physiology, 281(2):H559-H565, 2001.

[13] Dwain L Eckberg. Human sinus arrhythmia as an index of vagal cardiac outflow. Journal of Applied Physiology, 54(4):961-966, 1983.

[14] M Bolanos, H Nazeran, and E Haltiwanger. Comparison of heart rate variability signal features derived from electrocardiography and photoplethysmography in healthy individuals. In Engineering in Medicine and Biology Society, 2006. EMBS'06. 28th Annual International Conference of the IEEE, pages 4289-4294. New York City, 2006.

[15] Marco Di Rienzo, Emanuele Vaini, Barbara Bruno, Paolo Castiglioni, Prospero Lombardi, Gianfranco Parati, Carolina Lombardi, Paolo Meriggi, and Francesco Rizzo. Wearable Seismocardiography: Towards the beat-to-beat assessment of cardiac mechanics during sleep in microgravity. 2014 8th Conference of the European Study Group on Cardiovascular Oscillations, ESGCO 2014, pages 239-240, 2014.

[16] Marco Di Rienzo, Emanuele Vaini, Paolo Castiglioni, Prospero Lombardi, Paolo Meriggi, and Francesco Rizzo. A textile-based wearable system for the prolonged assessment of cardiac mechanics in daily life. In Engineering in Medicine and Biology Society (EMBC), 2014 36th Annual International Conference of the IEEE, pages 6896-6898. IEEE, 2014.

[17] J Ramos-Castro, J Moreno, H Miranda-Vidal, M a García-González, Mireya Fernández-Chimeno, G Rodas, and Ll Capdevila. Heart rate variability analysis using a seismocardiogram signal. Conference proceedings : Annual International Conference of the IEEE Engineering in Medicine and Biology Society. IEEE Engineering in Medicine and Biology Society. Conference, 2012:5642$5,2012$.

[18] Alexandre Laurin, Andrew Blaber, and Kouhyar Tavakolian. Seismocardiograms return valid heart rate variability indices. In Computing in Cardiology Conference, pages 413-416, 2013.

[19] Mojtaba Tadi, Eero Lehtonen, Tero Koivisto, Mikko Paukkunen, Ari Paasio, and Mika Teras. Seismocardiography : Toward Heart Rate Variability ( HRV ) Estimation. In IEEE International Symposium on Medical Measurements and Applications, 2015.

[20] Joonas Paalasmaa, Hannu Toivonen, and Markku Partinen. Adaptive heartbeat modelling for beat-to-beat heart rate measurement in ballistocardiograms. IEEE Journal of Biomedical and Health Informatics, 19(6):1-8, 2014.

[21] David Friedrich, Xavier L. Aubert, Hartmut Führ, and Andreas Brauers. Heart rate estimation on a beat-to-beat basis via ballistocardiography - A hybrid approach. 2010 Annual International Conference of the IEEE Engineering in Medicine and Biology Society, EMBC'10, pages 40484051, 2010.

[22] JH Shin, BH Choi, YG Lim, DU Jeong, and KS Park. Automatic ballistocardiogram (bcg) beat detection using a template matching approach. In Engineering in Medicine and Biology Society, 2008. EMBS 2008. 30th Annual International Conference of the IEEE, pages 1144-1146. IEEE, 2008.

[23] Jae Hyuk Shin, Su Hwan Hwang, Min Hye Chang, and Kwang Suk Park. Heart rate variability analysis using a ballistocardiogram during Valsalva manoeuvre and post exercise. Physiological measurement, 32(8):1239-1264, 2011.

[24] Christoph Brueser, Stefan Winter, and Steffen Leonhardt. Unsupervised heart rate variability estimation from ballistocardiograms. International Journal on Bioelectromagnetism, 15(1):1-6, 2013. 
[25] Eduardo Pinheiro, Octavian Postolache, and Pedro Girão. Blood pressure and heart rate variabilities estimation using ballistocardiography. In Proceedings of the 7th Conf. on. Telecom, pages 125-128, 2009.

[26] F Khosrow-Khavar, K Tavakolian, and C Menon. Moving toward automatic and standalone delineation of seismocardiogram signal. In Engineering in Medicine and Biology Society (EMBC), 2015 37th Annual International Conference of the IEEE, pages 7163-7166. IEEE, 2015.

[27] Paul M Stevens and Lawrence E Lamb. Effects of lower body negative pressure on the cardiovascular system. The American journal of cardiology, 16(4):506-515, 1965.

[28] William H Cooke, Kathy L Ryan, and Victor A Convertino. Lower body negative pressure as a model to study progression to acute hemorrhagic shock in humans. Journal of Applied Physiology, 96(4):1249-1261, 2004.

[29] V X Afonso, W J Tompkins, T Q Nguyen, and S Luo. ECG beat detection using filter banks. IEEE transactions on bio-medical engineering, 46(2):192-202, February 1999.

[30] F. Khosrow-khavar, K. Tavakolian, A. P. Blaber, J. M. Zanetti, R. Fazel-Rezai, and C. Menon. Automatic annotation of seismocardiogram with high-frequency precordial accelerations. IEEE Journal of Biomedical and Health Informatics, 19(4):1428-1434, July 2015.

[31] Peter D Welch. The use of fast Fourier transform for the estimation of power spectra: A method based on time averaging over short, modified periodograms. IEEE Transactions on audio and electroacoustics, 15(2):70-73, 1967.

[32] Jeffrey C Lagarias, James A Reeds, Margaret H Wright, and Paul E Wright. Convergence properties of the Nelder-Mead simplex method in low dimensionselder-mead simplex method in low dimensions. SIAM Journal on optimization, 9(1):112-147, 1998. 